\title{
Métaphores et analogies dans le discours des sciences de l'homme et de la société
}

Une introduction

Marie-Jeanne Borel

\section{OpenEdition}

Journals

Édition électronique

URL : http://journals.openedition.org/ress/704

DOI : $10.4000 /$ ress.704

ISSN : $1663-4446$

Éditeur

Librairie Droz

Édition imprimée

Date de publication : 1 février 2000

Pagination : $5-11$

ISBN : 2-600-00409-2

ISSN : 0048-8046

\section{Référence électronique}

Marie-Jeanne Borel, « Métaphores et analogies dans le discours des sciences de l'homme et de la société », Revue européenne des sciences sociales [En ligne], XXXVIII-117 | 2000, mis en ligne le 17 décembre 2009, consulté le 20 avril 2019. URL : http://journals.openedition.org/ress/704 ; DOI : $10.4000 /$ ress.704 
Marie-Jeanne BOREL

\title{
MÉTAPHORES ET ANALOGIES DANS LE DISCOURS DES SCIENCES DE L'HOMME ET DE LA SOCIÉTÉ Une introduction
}

\begin{abstract}
Depuis quatre ans, nous - le Groupe d'études qui s'est nommé lui-même, non sans prétention, «Raison et rationalités »- nous nous retrouvons à Bramois pour nous entretenir d'un thème pluridisciplinaire. Nous avons eu le souci de conserver un fil directeur, un problème-cadre capable de lier entre elles, dans leur variété et leurs couleurs, les contributions des participants dans la diversité de leurs disciplines propres. Il s'agit de la problématique des rationalités scientifiques, ou plus généralement de différents usages de la Raison dans les constructions scientifiques. Nous avons ainsi exploré des thèmes riches et multiples, qui ont à chaque fois débouché sur le suivant, et qu'indiquent les titres des numéros de la Revue Européenne des Sciences Sociales où ont paru les actes de nos rencontres: «Logique des lois» (1995), «De l'argumentation dans les sciences de la société » (1996), «Le changement dans les sciences de la société » (1997), enfin «L'image de l'homme dans les sciences de la société» (1998). C'est dans le prolongement de ces réflexions que nous avons imaginé celui de 1999; il s'agit des processus métaphoriques et des démarches analogiques à l'œuvre dans les discours de ces sciences, discours de preuve ou d'invention.

Lors de la dernière matinée du séminaire de 1998, les participants ont paru souhaiter continuer à tisser ce fil, mais en lui donnant un tour plus concret: qu'autour d'un objet commun (un épisode historique, une anecdote, une biographie), chaque discipline illustre dans sa pratique ses méthodes, comme un cuisinier préparant devant nos yeux ses recettes préférées. Une autre proposition a été de partir d'un grand mythe. On a parlé alors de celui du labyrinthe. La première proposition nous a paru prématurée: il nous a semblé en effet qu'il restait de ce fil comme une partie en amont, c'est-à-dire en amont de ce que la raison peut sciemment vouloir articuler et régler, et qu'il fallait peut-être se tourner vers ce qui la précède, en particulier sous cet avatar (de raison!) qu'indique le mythe du labyrinthe - dans l'interprétation qu'en a donné Friedrich Dürrenmatt - ce lieu où tout commence pour un chercheur, pauvre Minotaure qui ne sait pas de quel côté faire son premier pas, ni ce que reflète ce qu'il lui semble avoir vu. Cette métaphore nous a mis sur la piste de cet amont, et qui est précisément... la métaphore. Il s'agit d'une métaphore spatiale, de cheminements et de passages, il s'agit donc aussi du temps: un apprentissage de la symbolisation est mis en scène, qui s'achève avec la mise à mort du protagoniste, comme on sait. Voici l'histoire telle qu'elle est contée par Dürrenmatt.
\end{abstract}

- Le Minotaure est seul dans le labyrinthe, mais il n'a aucune idée de ce que «Minotaure», «seul» et «labyrinthe»veulent dire. Il n'a pas d' «idées», car il 
sent et il pense en images. Il ne connaît aucune des distinctions générales nécessaires à la connaissance d'un environnement humain (rêve/réalité, vie/mort, reflet/réel, un/deux, soilautre, etc.). Sa brève histoire est l'apprentissage de ce savoir.

- Les parois du labyrinthe sont des miroirs. Le Minotaure s'y voit réfléchi à l' infini, sans savoir ce qu'il voit. D'abord, il se voit autre, avant de se voir agent du mouvement des autres. Il joue alors de ce pouvoir, omnipotent. Soudain, voilà que l'un de ces autres ne joue pas le jeu; on voit cet être, mais sans pouvoir le toucher (où est-il?), et il agit pour son compte. En fait, c' est une fille que le Minotaure finit par attraper, son contact corporel le bouleverse; il la viole et la tue en une vague de désir fusionnel. Elle ne bouge plus, mais il rêve son mouvement.

- Un être entre dans son champ visuel. Il n'y a plus de reflets car le soleil brûle au zénith. Cet être ressemble à l'objet de son désir, sans être lui; mais le Minotaure en a souci, cette fois, car il veut le garder. Il ne perçoit pas le désir de tuer de l'autre. Blessé, il devient méfiant. Il joue alors un jeu de différences et de similitudes, et commence à imaginer des possibles, dont celui d'être menacé «du dehors». Fier de son bon droit «de dedans», il ressent haine et rage: il massacre tout sur son chemin, mais sans savoir d'où provient la menace.

- Quand plus rien ne bouge, il est tout seul avec ses images. Leur mime ridicule le rend fou, et il se jette aussi sur elles pour les détruire; mais l'autre qu' il voit hostile lui semble à la fois traître et insaisissable, et surtout étrange: "un autre pas comme les autres» (comme l'écrit René Zazzo). En fait, il n'y en a qu' un comme cela, et le Minotaure rêve cette fois qu' il est unique (un monstre) en même temps qu' un autre comme les autres.

- Endormi, voilà qu'Ariane lui noue en catimini un fil rouge autour des cornes. Puis voilà que s'approche un autre Minotaure (un autre monstre); il s'agit bien d'un autre et pas d'un reflet, car le Minotaure découvre de petites différences dans le mime, un petit retard rompant la simultanéité des gestes, une main gauche qui passe à droite à l'encontre de la symétrie du miroir et, surtout, chacun a ses propres reflets. Envahi par l'ivresse du «tu» fraternel, le Minotaure danse la fin du labyrinthe. Mais l' autre le tue : c'était un faux frère! Thésée ôte son masque de taureau et s'en va avec ses reflets, en rembobinant son fil rouge.

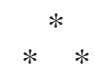

Le récit développe dans le temps l'expérience de l'altérité; il montre toute la difficulté d'en «tenir» une figuration qui rende l'espace vivable. Il existe deux conceptions (métaphores) de la direction du temps, donc de la pensée du futur dans le mouvement de la découverte. Pour l'une, on «va de l'avant» sans revenir sur ses traces, et on a alors son futur devant soi; pour l'autre, si on «voit devant», alors on ne voit que son passé (des archives, traces et textes), car le futur est, par définition, invisible! On voit que certaines idées chères à nos sciences (la Prévi- 
sion et la Prévention) n'ont ni la clarté ni la distinction d'idées de la Raison moderne. Il faut s'être appelé Platon pour avoir osé concevoir ce qui fait sens ainsi dans le temps sous l'aspect d'un seul et même mouvement: à la fois d'une part en avant (philosopher, c'est «apprendre à mourir», aller sans rien y voir devant), et d'autre part en arrière (philosopher, c'est «remémorer», revoir sans y aller). Pour le chercheur ordinaire en sciences humaines, ce double mouvement est vécu bien souvent avant de pouvoir être consciemment réfléchi, et peu de penseurs ont théorisé sciemment ce donné épistémologique - Hegel? Freud?

La métaphore du labyrinthe est risquée pour la réflexion épistémologique, non pas comme métaphore pour l'invention du savoir, mais à cause de ce qu'elle engage si on la prend pour seul guide, car on ne peut en tirer aucune méthode. On «tombe» dans une métaphore (comme dans une «potion magique»), de même qu'on «doit rester» dans un labyrinthe si on y est né, ou que l'histoire veut qu'on s'y trouve à un moment donné. On n'en ressort pas simplement «comme ça». Le Minotaure de Dürrenmatt, en fait, y meurt faute d'une capacité à « secondariser», c'est-à-dire faute de disposer d'une compétence sémiotique suffisante pour (1) se représenter soi-même comme un autre, donc anticiper ce que l'autre peut prévoir, et (2) se représenter l'autre comme soi et prévoir ce que l'autre peut anticiper. L'autre, ce n'est pas seulement l'autre humain avec qui parler, mais le monde où nous sommes quand nous en parlons! Ce monde, «temple où de vivants symboles laissent passer de confuses paroles », nous devons en faire partie pour pouvoir en dire quelque chose, mais sans pouvoir en être un élément lorsqu'on veut en parler. On ne sait (rien) de rien, si on n'est pas dehors, mais on ne sait rien (de rien ) si on reste dehors.

C'est pourquoi, en réfléchissant aux façons possibles d'aborder l'image du labyrinthe dans la perspective interdisciplinaire qui est la nôtre, nous avons retenu les idées de métaphore et d'analogie. Les modes de pensée métaphoriques sont en effet impliqués dans toute activité de modélisation, ou plus général encore, de schématisation, quand l'inconnu peut être saisi au moyen du connu grâce à des similitudes. Ils sont donc essentiels à toute démarche heuristique, dans l'invention des connaissances ordinaires et scientifiques, comme dans la communication rhétorique ou pédagogique. «Bien métaphoriser, c'est apercevoir le semblable», disait déjà Aristote, pour qui c'était aussi «transposer de l'anonyme dans un nom ». Identifier, c'est pour la pensée, le moyen de figurer les différences !

Laissons Ricœur en dire plus sur ce processus (1975, pp. 10-11): «La métaphore se présente comme une stratégie de discours qui, en préservant et développant la puissance créatrice du langage, préserve et développe le pouvoir heuristique développé par la fiction». Il y trouve le point d'articulation mobile entre l'organisation interne du sens et le pouvoir qu'ont pensée et langage de renvoyer hors de soi. Autrement dit, pour lui, par la métaphore, «le discours libère le pouvoir que certaines fictions comportent de redécrire la réalité». La métaphore comme re-description est un thème qui intéressera notre groupe.

L'analogie, quant à elle, a la métaphore pour fond, tout en étant davantage qu'une métaphore. De même que la comparaison, qui est déjà une métaphore déployée (n'en déplaise à Quintilien, qui pensait le contraire), elle en diffère en tant que développée comme un discours articulé en propositions qui s'enchaînent dans un certain ordre: l'analogie est un mode de raisonnement. On pourrait dire qu'il secondarise des significations que la métaphore lie de façon primaire 
(condense, déplace). Il y a une tradition en ce sens. C'est Théophraste en fait, le disciple d'Aristote suivant en cela son maître, qui a codifié le premier l'analogie comme une espèce de raisonnement, donc comme un élément de méthode (de logos). Ce raisonnement se sert du semblable comme d'une prémisse, prémisse que la métaphore aura inventée. Pour donner un exemple célèbre, il fallut à Harvey accomplir un acte assimilateur d'imagination intuitive pour «voir» une pompe à eau dans le fonctionnement d'un cœur vivant. Il s'en suivra toute une histoire, celle du développement méthodique de cette métaphore, par un pesage précis des similitudes et des différences et par la réalisation de modèles conceptuels autorisant l'inférence exacte de conséquences intéressantes; le bout de cette histoire se trouve aujourd'hui... en ingéniérie cardiologique.

On sait encore que ce mode de raisonnement peut se servir de différentes relations de comparaison, et qu'on aura de ce fait différentes sortes d'analogies. Certaines seront purement formelles, ou fonctionnelles, héritage de l'analogie appelée de «proportionnalité» depuis Boèce $(a / b=c / d$ est un rapport entre des rapports). On connaît son importance en musique et en arithmétique anciennes, mais l'analogie d'Harvey est aussi de ce type. La théologie s'est aussi servie d'analogies, mais qui sont dites de «proportion », car elles expriment ou sous-tendent davantage qu'un pur rapport de forme; par exemple, une sorte de bonté qui ressemble à la bonté divine peut s'appliquer à l'homme, car l'homme ressemble à Dieu en tant qu'il en est sa créature. On a un bel exemple du jeu de cette différence chez Platon, où ce que le Bien est dans le lieu intelligible pour l'intelligence et ses objets, le soleil l'est dans le lieu visible pour la vue et ses objets, d'une part (Timée, 31c), alors que d'autre part, le soleil est aussi «le rejeton que le Bien a engendré par analogie avec lui-même» (République, 6, 508b).

La pensée naturelle se sert largement de ces croyances en des liaisons substantielles (de genre, de cause, etc.) garantissant les ressemblances (soigner une brûlure à la jambe avec des cendres de jambe d'éléphant carbonisée). Or une des fonctions de la raison dans la science serait justement de renoncer à ces adhérences intuitives pour ne s'intéresser qu'aux aspects formels des mises en relation. En épistémologie, des doutes pèsent souvent sur le statut des analogies (illusion, béquille, échafaudage, etc.), un héritage d'Aristote, qui n'y voyait qu'un moyen de coordonner les genres (l'essence est au devenir ce que le savoir est à l'opinion), indispensable mais en dehors de la science comme telle. Il nous semble qu'on peut faire mieux...! Leibniz y voyait une capacité d'entre-expression des disciplines en chemin vers une science universelle, et Kant une activité régulatrice. Aujourd'hui, le raisonnement par analogie reste certes seulement «plausible» par contraste avec la déduction, mais on en reconnaît la portée irremplaçable pour l'étude des méthodologies de l'invention ou encore celles de l'expertise.

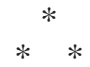

Il nous a paru fécond de réféchir aux relations entre métaphore, temps et espace dans la pratique de recherche en sciences humaines, et d'occuper le colloque de 1999 à méditer sur quelques thèmes possibles:

- l'espace utilisé comme métaphore pour se représenter des réalités non spatiales, notamment temporelles; 
- les métaphores produites pour figurer des expériences ou des descriptions de l'espace;

- les arguments et les raisonnements par analogie se servant de métaphores spatiales, leurs justifications méthodologiques et leur pouvoir heuristique dans divers contextes de discours;

- les logiques de la métaphore et de l'inférence analogique à différents niveaux d'élaboration rationnelle du discours scientifique;

- leurs usages dans la vie quotidienne vs dans la pensée scientifique;

- leurs essais de formalisation dans des logiques non-standard, par exemple en Artificial Intelligence;

- les usages qu'on peut trouver de ces figures et procédures dans l'histoire d'une discipline.

On pense ici à Bachelard notamment, surtout aux thèmes de sa Poétique de l'espace: l'intérieur et l'extérieur, le grand et le petit, l'ouvert et le fermé, l'intime, le caché et l'exhibé, le rond, etc.

Les contributions qu'on va lire sont variées. Le thème de la métaphore et de l'analogie est abordé du point de vue de différentes disciplines scientifiques, avec différents enjeux - historiques, épistémologiques ou encore critiques. L'«herbier » des exemples soumis à l'analyse est lui-même riche. Et même si, dans l'ensemble, les auteurs s'accordent sur la fonction poiétique des processus métaphoriques dans la production de la connaissance, et sur le pouvoir schématisant des constructions symboliques qui les réalisent, on insiste aussi, ici et là, sur d'autres propriétés - heuristiques, rhétoriques ou enfin idéologiques.

En répertoriant les principaux modèles de métaphores et d'analogies utilisés par la sociologie, Giovanni Busino montre à quel point il n'a pas été possible de s'en passer: empruntant à la physique, à la biologie, à l'économie, à la linguistique, à la sociologie elle-même par généralisation d'approches particulières, les sociologues ont parfois inventé, et se sont parfois égarés. Le questionnement de l'usage de ces modèles est en lui-même un «chantier» permanent, comme la sociologie elle-même.

Jean-Jacques Wunenburger met en lumière la nature ambivalente des opérations métaphorisantes. Partant de Platon et de son usage des paradigmes - servant à la saisie progressive de l'essence, par leur interprétation réflexive —, il montre les rapports étroits que la pensée nourrit avec les images spatiales et l'importance d'une rationalité imaginative à laquelle l'époque moderne a rendu le crédit: logos verbo-iconique, à pratiquer sans mépris et sans euphorie.

Sabine Prokhoris nous fait réfléchir à la psychanalyse comme «art des passages » en commentant l'énoncé elliptique de Freud à la fin de sa vie: «Psychè est étendue. N'en sait rien ». Il sert de guide pour une traque du métaphorique dans les relations du psychisme et du sens, de l'œuvre d'art et de la théorie. On revient ainsi sur l'après-coup, sur l'intime et son contraire, sur l'inconscient, l'innomé, le «discours de l'autre», puis sur le cadre analytique et l'amour de transfert. Tout pensée est métaphorique si l'écart et l'inadéquation sont sa vie même, et donc l'arrêt et la sclérose, sa mort. 
Opposant l'implosion sémantique qui caractérise la métaphore poétique à la fonction heuristique du raisonnement analogique dans les sciences historiques, Jean-Claude Passeron montre la différence de leurs procédures et de leurs effets. Contrôlée par la méthode comparative, l'analogie ouvre à l'observation le chemin d'un enrichissement indéfini des descriptions et des concepts sociologiques: lorsque la métaphore rencontre, dans l'observation des «séries», des contreexemples qui la réfutent, cet échec rhétorique augmente la connaissance scientifique tant du «comparant» que du «comparé».

Gérard Timsit prend la métaphore dans le discours juridique sous deux aspects, l'un figural, du transfert de sens d'un domaine à l'autre, l'autre, structural, générateur de forme, donc outil essentiel de la compréhension (rendre visible ce qu'on n'en voit pas). Il nous montre leur capacité schématisante dans deux lieux d'écriture, le discours du droit et le discours sur le droit, et l'intérêt de cette étude pour la critique du raisonnement juridique.

Pierre Moor nous engage dans un paradoxe, celui du labyrinthe: de quoi parle au fond une métaphore, quand on sait que plus elle est claire, donc donne accès à ce qu'elle vise, plus elle est fausse, car donnant cet accès sous une forme seconde. Comment sait-on de quoi elle parle, qu'elle ne dit pas, et que dit-elle de celui qui en use? Devoir métaphoriser quand on ne peut pas calculer, c'est aussi le lot du discours du droit. On voit comment la question de la densité normative se ramène inévitablement à des jeux hétérogènes et variés entre contrainte et liberté, systèmes et applications. La loi est en soi une métaphore - comme Gérard Timsit l'a montré - (il n'y a pas de syllogisme qui vaille !) qui montre toujours, derrière son objet, quelque désir soit de pouvoir soit de liberté.

C'est aux analogies du réseau - des villes et des pouvoirs - que Denise Pumain et Jean-Pierre Gaudin consacrent leur étude, avec la question du devenir abstrait d'une métaphore d'abord concrète. A partir des années 60, ils explorent diverses configurations de discours et d'enjeux associés, au moyen desquels s'affrontent d'une part une problématique de la contrainte (parcours obligés, déplacements organisés, hiérarchisés), d'autre part une problématique plus empirique de la complexité sociale. Aujourd'hui, ce sont de nouvelles métaphores naturalistes qui s'imposent en une vision «new age» fusionnant réseaux neuronaux et informationnels.

Claude Mouchot donne de très fines analyses critiques, fort ironiques, de l'ontologie sous-jacente que le discours économique néo-classique véhicule dans ses analogies de l'équilibre inspirées de la physique. Il montre comment certaines sont seulement formelles, d'autres confuses, d'autres imposées à une réalité économique qui n'en demande pas tant. La métaphore de l'équilibre général paraît être la plus rhétorique. Le problème central ainsi posé est que toutes ces métaphores échouent à saisir ce qu'elles prétendent: saisir effectivement quelque chose de la dynamique économique. La cause réside dans l'échec à réduire le temps de l'action et le temps vécu au paramètre des physiciens. Sont-ils théorisables? Oui si l'on admet que la modélisation rationnelle doit commencer par être raisonnable, sa condition sine qua non.

Henri Volken choisit l'histoire des mathématiques pour montrer comment une métaphore floue, celle d'infini, vieille de 2000 ans, ne s'est nullement épuisée depuis le temps, même (et surtout pas) après Cantor, Hilbert et Gödel, nos quasicontemporains. Il montre très simplement comment la construction linéaire, pas à 
pas, des nombres naturels, de laquelle semble exclu tout mystère, offre en réalité des paradoxes redoutables quant à l'infini, car bien que la méthode soit toute transparente, il se trouve des nombres imaginables mais inaccessibles par elle, ou inversement, des entités inimaginables, mais qu'on sait construire. Actuel, potentiel, la nature de l'infini reste un problème.

Jacques Coenen-Huther s'intéresse à l'histoire des rapports conflictuels entre sociologie et géographie, mais à la recherche de facteurs d'interdépendance. Il trouve ceux-ci dans certaines métaphores intégrant abstractions relationnelles et espaces de vie, comme celle d'organisme ou de lieu familier. Les deux disciplines vont ainsi faire se «croiser» des problématiques comme celle des limites.

Enfin, Hervé Le Bras illustre par l'analyse d'œuvres de Kafka le jeu des différences dans la pluralité de temporalités diverses et la restitution qui en est faite par le choix opéré par celui qui décrit cette pluralité. De même pour l'espace. Temps et espaces sont ainsi recréés sous une forme qui est celle de labyrinthes, rapprochant ce qui est éloigné et séparant ce qui est voisin

\section{Université de Lausanne}

\section{QUELQUES RÉFÉRENCES}

Bachelard Gaston, La poétique de l'espace, Paris, PUF, $7^{\mathrm{e}}$ éd., 1997.

Canguilhem Georges, Modèles et métaphores dans la découverte en biologie, in Etudes d'histoire et de philosophie des sciences, Paris, Vrin, 1968 (trad. Michel Leyvraz et Jean-Paul Clerc).

Dürrenmatt Friedrich, La mort de la Pythie, suivi de Minotaure, Lausanne, L'Age d'Homme, 1990. Lakoff Georges, Les métaphores dans la vie quotidienne (Metaphores we live by), trad. M. Defornel, Paris, Minuit, 1985.

Goodmann Nelson, Manières de faire des mondes, Paris, Chambon, 1992.

Hesse Mary B., Models and Metaphors in Science, New York, 1963.

Ricœur Paul, La métaphore vive, Paris, Seuil, 1975

Zazzo René, Reflets de miroir et autres doubles, Paris, PUF, 1993. 